\title{
LES MÉTAPHORES DES TEXTES BOURSIERS
}

\author{
Sandra Rodriguez et Aurore Van de Winkel ${ }^{1}$
}

Qu'est ce donc que la Bourse? N'importe quel dictionnaire nous en donnera une définition de type : "Marché public organisé où s'effectuent des transactions sur des valeurs, des marchandises ou des services; le lieu où se réunissent les personnes qui interviennent sur ce marché $»^{2}$. Pourtant peu d'informations concrètes sur le fonctionnement de cette institution semblent être accessibles au grand public. Si ce dernier veut de plus amples renseignements, il devra les chercher dans des livres consacrés à ce sujet, des sites internet spécialisés et plus généralement dans les textes journalistiques, relatifs à l'information boursière, disponibles dans les revues ou les journaux. Or il n'est pas dit que ces documents permettent aux lecteurs de bien comprendre cette institution.

Comment ces textes journalistiques parlent-ils de la Bourse et de son fonctionnement ? Comment les médias scripto-visuels rendent-ils compte de la réalité boursière et comment arrivent-ils à orienter les représentations des lecteurs? Quelles sont les représentations qu'intègrent véritablement ces derniers ? Que comprennent-ils à ce phénomène après la lecture de ces articles ?

1 Sandra Rodriguez et Aurore Van de Winkel sont étudiantes du DEA au Département de communication de l'UCL.

2 J. REY-DEBOVE, A. RAY, sous la dir. de, Le Petit Robert, Paris, Dictionnaires Le Robert, 1996, p. 252.

Recherches en communication, $\mathrm{n}^{\circ} 23$ (2005). 
C'est à ces questions que nous allons tenter de répondre dans l'article suivant. Nous allons plus particulièrement nous pencher sur les métaphores et les expressions métaphoriques, présentes dans les articles de presse traitant de la Bourse, qui nous aideront à comprendre la façon dont cette institution est décrite et pourrait être comprise par le lecteur.

Selon G. Lakoff, la métaphore est bien plus qu'un jeu linguistique ou qu'une figure de rhétorique parmi d'autres : elle est un instrument majeur de la pensée ${ }^{1}$. En effet, ces images verbales, liées à la vie courante et appliquées à un objet particulier, peuvent orienter l'imaginaire rattaché à ce dernier et aider ainsi à sa compréhension. Elles permettent de relier un savoir nouveau à un savoir déjà intégré par la projection des qualités d'un objet sur un autre. L'étude des métaphores et des expressions métaphoriques des textes boursiers pourra donc nous amener à tirer des conclusions intéressantes sur la façon dont est présentée la Bourse dans les textes journalistiques.

Nous sommes parties d'un corpus constitué de huit articles de presse économiques spécialisés et de huit autres non spécialisés dans lequel nous avons tenté de relever les différentes métaphores et expressions métaphoriques utilisées. Nous avons ensuite regroupé celles-ci par thèmes et nous les avons classées selon leur fréquence d'apparition dans les textes. À partir de ces résultats, nous avons étudié les différents thèmes métaphoriques présents, l'image et les qualités qu'ils attribuaient à la Bourse et leurs rôles dans la compréhension du fonctionnement de cette institution.

\section{Les articles de presse économiques non spécialisés et leurs métaphores}

Dans les articles de presse économiques non spécialisés, nous avons relevé treize sortes de métaphores ou expressions métaphoriques différentes.

Les personnifications ou l'anthropomorphisation d'entités abstraites et d' "objets» liés à la Bourse sont les plus fréquentes. En effet, la totalité des articles attribuent des propriétés, des actions humaines soit à cette institution, aux sociétés ou aux entreprises qui y

1 G. PIROTTON, «Métaphore et communication pédagogique. Vers un usage délibéré de la métaphore à des fins pédagogiques », Recherches en communication, n², 1994, p. 75. 
sont cotées..., soit aux actions, titres, devises, cours et autres « objets » liés à son fonctionnement. Ainsi le texte "Intéressants, les cliquets?» parle de la «durée de vie de la SICAV» tandis que l'article intitulé «Les warrants ref(er)ont surface » explique que «le marché belge des warrants a manqué une étape importante de son développement ».

Dans les huit articles, sont apparus à six reprises des métaphores de course sportive et de performance. Le texte "Le Nasdaq contre le Dow Jones » utilise, par exemple, des expressions comme «battre son record», "performance honorable » ou encore «accrochez vos ceintures!». Dans l'article «Intéressants, les cliquets? », les termes " départ et arrivée », « en fin de parcours », « déjouer les pièges », etc. relèvent également de ce thème métaphorique.

Les expressions métaphoriques concernant la gloire, le succès et le vedettariat, telles que "stars de la nouvelle économie », "engouement », " coqueluches », etc. ${ }^{1}$, apparaissent dans quatre textes comme les métaphores de mouvement exprimées notamment par des mots comme "frémissement», "croissance négative», "mouvement inverse », etc. ${ }^{2}$. En cinquième position, nous trouvons les expressions métaphoriques concernant le risque, la stratégie, le choix ( «pari audacieux $»$, etc. $^{3}$ ). Elles apparaissent dans trois articles comme celles relatives au conflit et à la guerre. L'auteur du texte «Acheter au son du canon? ? utilise ainsi les expressions suivantes: «son $d u$ canon», « son du clairon », « victimes », « armistice », etc. ${ }^{4}$.

Les métaphores sur le jeu reviennent à deux reprises, comme celles de l'aviation ou du «meeting » aérien. Les expressions métaphoriques sur l'image/le reflet ( tel un miroir $»^{5}$, etc.) et celles du domaine financier sont également présentes deux fois. Enfin celles concernant la météorologie ( «adapté à la météo boursière »", etc.), les sentiments et la mer (« creux de la vague $\left.»^{7} . ..\right)$ n'apparaissent qu'une seule fois.

1 D. DAOÛT, «Le Nasdaq contre le Dow Jones », Le Vif/L’Express, 3 mars 2000, p.118.

2 ID., «Acheter au son du canon ?», Le Vif/L'Express, 26 janvier 2001, pp.96-97.

Ibid.

Ibid.

ID. «Le Nasdaq contre le Dow Jones », Le Vif/L'Express, 3 mars 2000, p.118.

P.V.C., « Les warrants ref(er)ont surface », La Libre Belgique, 16 mai 2001, p.21.

7 D. DAOÛT, «Acheter au son du canon? », Le Vif/L'Express, 26 janvier 2001, pp.96-97. 


\section{Les articles de presse économique spécialisés et leurs méta- phores}

Comme dans les articles de presse économiques non spécialisés, c'est la personnification d'entités abstraites et d' "objets » relatifs à la Bourse qui apparaît le plus fréquemment puisque ce procédé est présent dans les huit textes ( "la déprime des marchés boursiers », «les valeurs délaissées $»^{1}$, etc.). Nous retrouvons, à six reprises, les expressions métaphoriques concernant la compétition sportive, la course ou la performance sous la forme notamment de formules telles qu' "améliorer leur performance $»^{2}$, «meilleurs scores $»^{3}$, etc. La métaphore de la maladie et de la dépression ( «souffrir du ralentissement », «causer $d u$ souci », «ambitions handicapées ${ }^{4}$, etc.) arrive en troisième position en apparaissant dans trois textes comme celle de la guerre, de la révolte et des conflits («artillerie lourde», «tir groupé », « revendiquer $»^{5}$, etc.).

Les thèmes métaphoriques des expressions juridiques, de la séduction, de la hiérarchie - que l'on retrouve dans les mots «position», «le géant allemand $»^{6}$, etc. -, de la stratégie/du jeu ("parier», «échiquier $»^{7}$, etc.) et du domaine maritime («en amont», «en aval $»^{8}$, «la décrue $»^{9}$, etc.) ne sont toutes présentes que deux fois.

Enfin, nous n'avons relevé qu'à une seule reprise les expressions métaphoriques relatives au domaine aérien («volatilité »10, etc.), aux catastrophes naturelles («raz-de-marée », «déferlante Interbrew» ${ }^{11}$,

1 Auteur anonyme, «Une année de perdue pour les fonds de pension : le return moyen s'élève à $-0.1 \%$ en 2000 », L'Echo, 29 mars 2001.

2 Auteur anonyme, «La révolte des actionnaires », Tendances, 24 mai 2001,p.46.

3 Auteur anonyme, «Une année de perdue pour les fonds de pension : le return moyen s'élève à $-0.1 \%$ en 2000 », L'Echo, 29 mars 2001.

4 A.D., « Belle croissance bénéficiaire en perspective pour les distributeurs alimentaires européens », L'Echo, 7 juin 2001.

5 P. VAN CAMPENHOUT, « Brokers online en panne », La Libre Belgique, 10 mars, 2001, pp.4-5.

6 Auteur anonyme, «La révolte des actionnaires », Tendances, 24 mai 2001,p.46.

7 V. SLITS, «Petit parfum spéculatif en ce début d'été », La Libre Belgique, 30 juin 2001.

8 A.D., op cit.

9 Auteur anonyme, «Une année de perdue pour les fonds de pension : le return moyen s'élève à $-0.1 \%$ en 2000 », L'Echo, 29 mars 2001.

10 Auteur anonyme, «La révolte des actionnaires », Tendances, 24 mai 2001,p.46.

11 M. SEPHIHA, «La présence d'Interbrew chamboule les statistiques d'Euronext 
etc.), à l'agriculture, aux transactions financières, à la météorologie (« détérioration sensible du climat », « l'horizon s'assombrit $»^{1}$, etc.), à la machine en panne ainsi qu'au mouvement.

Ainsi dix-sept thèmes métaphoriques ont été trouvés dans les articles de presse économiques spécialisés.

\section{L'anthropomorphisation ou l'expression métaphorique la plus utilisée}

L'une des structures métaphoriques les plus utilisées qu'il est possible d'observer est donc celle de la personnification ou de l'anthropomorphisation. Nous la trouvons dans la totalité des textes du corpus analysé qu'il s'agisse d'articles de presse économiques spécialisés ou non spécialisés. Par ce procédé, les auteurs donnent un caractère humain aux différents acteurs liés à cette institution. Cela comprend la Bourse elle-même mais aussi les entreprises, les brokers, le marché... Les «objets » propres à ce domaine boursier - soit les actions, les obligations, les valeurs, les warrants, les SICAV à cliquets, etc. - sont aussi personnifiés. Une valeur sera, par exemple, « déprimée », « abandonnée » ou, au contraire, «victorieuse ».

D'une certaine manière, cette anthropomorphisation rend concret et donc plus abordable le phénomène boursier. Il permet au lecteur de définir les acteurs et les objets de la Bourse ainsi que de clarifier leurs rôles en leur donnant une «personnalité ». Puisqu'il est naturellement plus facile de comprendre les réactions d'une personne que celles d'une institution, d'une entité abstraite, la personnification permet dès lors de simplifier la compréhension des relations qu'entretiennent les différents acteurs boursiers. Mais qui dit «personnalité » dit aussi « caractère ». Et la personnification agit également sur l'image que le lecteur se fera de ces acteurs.

Puisque les «personnages » boursiers sont ainsi identifiés, il devient plus facile de les placer dans un contexte ou un univers métaphorique, qui viendra définir d'avantage les relations existant entre eux. Dès lors, tout nous permettrait de croire que le choix des métaphores possibles pour illustrer les phénomènes boursiers serait des plus variés. Or chose étrange, dans les deux types d'articles du corpus analysé, nous

Bruxelles », L'Echo, 30 mai 2001, p.1.

1 P. VAN CAMPENHOUT, « Nasdaq un jeudi noir teinté de vert », La Libre Belgique, 24 mars 2001. 
retrouvons les mêmes sources d'inspiration métaphorique ainsi que des recoupements dans les champs de représentation utilisés.

L'anthropomorphisation est plus un style métaphorique qu'une métaphore spécifique. Tout en permettant l'identification et la projection des lecteurs aux acteurs boursiers, la personnification donne une image, une représentation plus humaine et concrète de la Bourse aux néophytes tout en maintenant l'image qu'ont déjà les initiés de cette institution.

\section{Comparaison de l'utilisation des métaphores dans les deux sortes d'articles}

Grâce à cette recherche, nous avons pu déceler des différences entre les textes de presse économiques spécialisés et les non spécialisés dans leur utilisation des métaphores.

En effet, d'un premier abord, la presse non spécialisée semble utiliser moins de métaphores que la presse spécialisée. Mais si l'on regarde les résultats plus attentivement, il semblerait que la première utilise plus souvent les mêmes métaphores pour décrire la Bourse et son fonctionnement. Elle a tendance à approfondir ces thèmes métaphoriques en utilisant de nombreuses expressions qui y sont relatives. Inversement, la presse spécialisée emploie de nombreuses métaphores différentes mais isolées. Ces dernières ne sont en effet présentes que par le biais de quelques mots s'y rapportant. Peu d'entre elles construisent vraiment l'ensemble du récit.

À ce sujet, nous pouvons proposer une hypothèse qui expliquerait cette différence. La presse spécialisée s'adresse, en effet, à un public de néophytes, ne connaissant que très peu le monde boursier. Pour ne pas l'embrouiller avec une description détaillée du fonctionnement complexe de cette institution, les journalistes sélectionnent quelques métaphores clés qui donnent des images plus générales de cette institution mais aucune d'entre elles n'est globale. La presse spécialisée s'adresse, elle, à un public de spécialistes, de financiers. Elle est donc tenue de parler de la Bourse de la façon la plus précise et la plus conforme à la réalité possible. Une seule métaphore ne permettrait pas de mettre en évidence tous les aspects saillants de cette institution. C'est pourquoi les journalistes ont recours à des multiples expressions métaphoriques peu développées. De plus, ces dernières ne s'attachent plus à donner une image générale de la Bourse mais une image précise de toutes ses composantes. 


\section{Les métaphores et leurs rôles}

Nous l'avons vu : les métaphores sont fortement utilisées dans les articles de presse économiques traitant de la Bourse. Ceci est dû en partie à la spécificité même des textes journalistiques qui sont destinés à un public large et non-scientifique. Les auteurs se permettent alors de vulgariser certaines informations en y glissant des métaphores. La théorie de Caroline Huynen et de Marc Lits, qui considère que le lecteur de textes journalistiques cherche dans les métaphores qui s'y trouvent "moins des effets de style qu'une synthèse informative et plutôt transparente de l'événement ${ }^{1}$, illustre bien le rôle d'une grande majorité des métaphores utilisées dans le corpus. En effet, ces dernières permettent de mettre en évidence rapidement et facilement un aspect de la Bourse qui serait difficile à développer de manière littérale.

À cause de la complexité et du haut degré d'abstraction du sujet abordé, ces métaphores, reprenant des phénomènes familiers et connus, simplifient le message et les informations diffusés. De plus, nous avons pu constater qu' aucun journaliste n'a pu trouver une métaphore unique permettant de représenter totalement l'institution. Le recours aux vingt thèmes métaphoriques n'a, en effet, permis de mettre en exergue que trois des caractéristiques de la Bourse. Nous développerons ce point ultérieurement.

Une autre sorte de métaphores présentes est ce que nous pouvons appeler les métaphores d'usage. Celles-ci sont fort présentes dans notre corpus et ont pour fonction de stabiliser la réception du texte en reproduisant un discours déjà socialement intégré ${ }^{2}$. Ce sont des «métaphores conventionnelles (...) [qui] structurent le système conceptuel ordinaire de notre culture qui est reflété dans notre langage quotidien $»^{3}$. Ces métaphores ont intégré le langage et les modes de pensée. Elles sont devenues courantes et ont perdu une partie de leur caractère métaphorique par leur littéralisation. Ce sont, par exemple, les expressions métaphoriques provenant du domaine juridique, retrouvées à quatre reprises dans le corpus.

1 C. HUYNEN, M. LITS, «La métaphore dans la presse écrite », Recherches en communication, $\mathrm{n}^{\circ} 2,1994, \mathrm{p} .49$.

2 Ibid., p.47.

3 G. LAKOFF, M. JOHNSON, Les métaphores de la vie quotidienne, Paris, Éditions de Minuit, coll. Propositions, 1985, p. 13. 
Les métaphores peuvent être également utilisées pour masquer un manque de compréhension du phénomène boursier par les journalistes eux-mêmes. Nous pensons ici plus particulièrement aux auteurs des textes journalistiques non spécialisés qui peuvent éviter de rentrer dans des détails qu'ils ne maîtrisent peut-être pas eux-mêmes.

Les métaphores ont également une visée relationnelle. Elles cherchent à accrocher l'attention du lecteur et à instaurer une relation de complicité due à l'utilisation d'horizons de référence communs. C'est la fonction des expressions métaphoriques présentes dans les titres et le chapeau des articles, généralement lus en premier par les lecteurs.

Les métaphores présentes ont donc plusieurs rôles. Elles peuvent aider à la compréhension d'aspects particuliers de la Bourse. Selon le cas, elles dramatiseront les échecs des acteurs boursiers ou valoriseront leurs résultats performants. Par contre, elles ne donnent que très rarement une image générale de cette institution; ce n'est le cas que de la métaphore de la machine qui n'est présente qu'une seule fois dans le corpus. Elles peuvent également illustrer un propos, « colorer» les textes par des expressions courantes ou encore accrocher le lecteur.

\section{Les métaphores et leur impact sur la compréhension de la Bourse}

Nous retrouvons, dans les deux types de textes étudiés, des métaphores très semblables qui visent à mettre en évidence certains aspects spécifiques de la Bourse. Ces figures de style apportent, en effet, un éclairage intéressant sur trois caractéristiques particulières de cette institution et de son fonctionnement.

L'imprévisibilité des cours des actions, des titres et des obligations est la première caractéristique de la Bourse que nous pouvons retrouver dans ce corpus. Elle est expliquée par les métaphores relatives aux catastrophes naturelles, aux courants marins, à la météorologie et à la panne, sujets connus couramment pour leur caractère imprévisible. Présente à une seule reprise dans la presse spécialisée, nous pouvons faire l'hypothèse que cet aspect est occulté au profit de la troisième caractéristique de la Bourse dont nous parlerons ci-dessous.

La deuxième caractéristique de la Bourse mise en exergue est celle relative aux fluctuations, aux mouvements perpétuels du cours des actions, des obligations et des titres. Celles-ci sont présentes sous la forme d'expressions métaphoriques relatives à la dépression/la maladie, à la hiérarchie, aux transactions financières, à l'aérien, au maritime 
et au mouvement. Ces thèmes reviennent neuf fois dans les textes de presse non spécialisés et six fois dans les spécialisés. Il est intéressant de constater que la presse non spécialisée utilise six métaphores différentes pour désigner ce phénomène contre trois dans la spécialisée.

La troisième caractéristique et la plus importante est le caractère stratégique des actes entrepris par les différents acteurs (les banques, les sociétés de bourse, les entreprises, les particuliers, etc.). Cet aspect est celui qui est le plus fortement et le plus souvent mis en évidence grâce aux métaphores de la compétition, de la course sportive, de la guerre, de la séduction, du jeu, du risque ou encore du «meeting » aérien. Ces figures de style apparaissent quatorze fois dans les articles de presse spécialisés et vingt fois dans les non spécialisés. Cette particularité semble donc, pour les auteurs, plus « essentielle » à diffuser auprès du public néophyte.

La compétition stratégique sous forme de course sportive est la plus souvent utilisée. Elle place les acteurs boursiers dans le rôle des joueurs qui doivent développer des stratégies pour pouvoir réussir. Or la course rappelle aussi deux notions essentielles : celle de la concurrence et celle de la performance. En effet, le but premier d'une compétition est d'être le gagnant de la course, celui à qui revient la gloire, le succès et implicitement l'argent. Le vainqueur est valorisé par les expressions métaphoriques extrêmes du succès, de la gloire et du vedettariat, la situation des perdants est, elle, dramatisée par les thèmes de la défaite, presque de l'anéantissement.

La course rappelle aussi la notion de rapidité, ce qui indique que placer en bourse n'est pas un acte qui s'évalue à long terme mais un jeu dangereux dans lequel les réflexes doivent être rapides. Cette métaphore nous permet de croire que l'essentiel n'est pas de comprendre en détail le fonctionnement interne de la Bourse mais de savoir comment $\mathrm{y}$ agir et ce, au plus vite. Cela donne l'impression au lecteur qu'il vaut donc mieux ne pas réfléchir et suivre les conseils des journalistes à la lettre sans trop se poser de questions.

L'importance de la métaphore de la course est peut-être également due au fait qu'elle permet de parler accessoirement des deux autres caractéristiques de l'institution : la fluctuation, représentée par le mouvement des participants ainsi que de leurs actions, et l'imprévisibilité, liée aux résultats incertains de la compétition.

L'importance du thème de la stratégie, de la compétition et plus spécifiquement de la performance nous ramène à la notion de positionnement du lecteur. En effet, les différentes métaphores arrivent à 
donner une certaine image du fonctionnement interne non pas de la Bourse mais de ses composantes. Or le lecteur, même s'il a un statut de spectateur, est invité, par les métaphores, à s'identifier aux acteurs boursiers, à être l'une de ces composantes. Il doit apprendre comme les " champions », et donc comme les investisseurs, à évaluer le parcours, les obstacles, les risques afin d'envisager un certain nombre de stratégies qui lui permettront de devenir gagnant ou bien de perdre (ce qu'il ne souhaite évidemment pas).

\section{Les métaphores utilisées sont-elles appropriées pour parler de la Bourse?}

Une métaphore repose sur deux similarités : la similarité interdomaine (c'est-à-dire qu'elle compare des objets provenant de deux domaines différents) et la similarité intradomaine (c'est-à-dire l'isomorphisme des structures, la similarité de la place qu'occupent ces objets à l'intérieur de leur domaine respectif). Selon Philippe Verhaegen ${ }^{1}$, pour qu'une métaphore fonctionne, il faut une faible similarité interdomaine et une forte similarité intradomaine. Il semblerait que la grosse majorité des thèmes métaphoriques retenus, c'est-à-dire ceux qui sont représentés par deux mots et plus, a ces qualités.

Mais nous devons mettre un bémol à ce résultat. En effet, sur les trois caractéristiques de la Bourse mises en évidence par les expressions métaphoriques, la plus développée est donc celle relative aux comportements des acteurs, à leurs plans stratégiques. Les deux autres aspects sont, elles, liées au « comportement » des objets des transactions de ces acteurs (les actions, titres...).

Les métaphores permettent donc de comprendre comment les acteurs interagissent, que les cours évoluent rapidement, etc. mais pas de comprendre globalement et concrètement ce qu'est la Bourse, quel est son but, comment fonctionne-t-elle, pourquoi les cours réagissentils de cette façon.

Le phénomène boursier reste donc relativement incompris et le lecteur ne devient pas spécialiste de la Bourse en ne lisant que les articles de presse sur le sujet. Il devra compléter ces renseignements par d'autres sources d'informations.

$1 \mathrm{Ph}$. VERHAEGEN, cours de «Vulgarisation scientifique : théorie et étude de cas », Louvain-la-Neuve, UCL, 2000. 


\section{En guise de conclusion}

La Bourse est donc un phénomène complexe qui ne semble pas être clarifié par l'utilisation de métaphores dans les textes journalistiques. Que ce soit dans les articles de presse spécialisés ou non spécialisés, les journalistes maintiennent une certaine incompréhension du phénomène et de son fonctionnement. Ils nous en donnent une représentation éclatée et incomplète et certains aspects importants de ce mécanisme semblent être occultés. Pourquoi cette opacité ? Peut-on supposer qu'il existe une véritable volonté de la part des auteurs des textes journalistiques de cacher aux lecteurs le véritable fonctionnement boursier? Ou les journalistes ne supposent-ils pas tout simplement que la compréhension concrète de l'institution intéresse moins le public que des conseils précis pour «faire de l'argent»? L'avis de spécialistes du domaine boursier ainsi que des auteurs de ces textes boursiers permettrait de vérifier les hypothèses émises au cours de cet article et apporterait également des éléments de réponses à ces questions laissées en suspens.

Quoi qu'il en soit, les métaphores ont toutefois leur utilité et sont fort présentes dans notre corpus. Elles donnent l'impression d'être une aide à la compréhension. En fait, elles allègent et « colorent » le propos par des expressions provenant soit du langage courant, soit du langage boursier. Elles mettent également en valeur quelques aspects intéressants de la Bourse par la projection de domaines déjà connus.

Nous pouvons conclure que les savoirs informels de type journalistiques sur le sujet de la Bourse nous donnent tout de même, par le biais des métaphores, une image complexe mais partielle et caricaturale de cette institution. Les personnes voulant mieux connaître ce phénomène ne peuvent se contenter de l'information disponible dans les textes journalistiques et devront compléter leur savoir sur le sujet par d'autres sources d'informations et d'explications peut-être plus formelles.

Peut-être pourront-elles alors savoir comment agir dans la Bourse et interagir avec ses acteurs en vue de bien gérer leurs avoirs dans cet univers où « rien n'est jamais assuré et aucun investissement boursier $n^{\prime}$ est garantit sans risque $»^{1}$.

1 D. DAOUT, «Acheter au son du canon? », Le Vif/L'Express, 26 janvier 2001, p. 96. 
